\title{
Application of a standardised protocol for hepatic venous pressure gradient measurement improves quality of readings and facilitates reduction of variceal bleeding in cirrhotics
}

Tze Tong Tey ${ }^{1}$, mBBS, MRCP, Apoorva Gogna ${ }^{2}$, MBBS, FRCR, Farah Gillan Irani², MBBS, FRCR, Chow Wei Too ${ }^{2}$, MBBS, FRCR, Hoau Gong Richard $\underline{L O}^{2}$, MBBS, FRCR, Bien Soo $\underline{\operatorname{Tan}^{2,3}}$, MBBS, FRCR, Kiang Hiong $\underline{\operatorname{Tay}^{2,3}}$, MBBS, FRCR, Hock Foong Lui ${ }^{4}$, MBBS, MRCP, Pik Eu Jason $\underline{\text { Chang }}{ }^{1,3}$, MBBS, MRCP

INTRODUCTION Hepatic venous pressure gradient (HVPG) measurement is recommended for prognostic and therapeutic indications in centres with adequate resources and expertise. Our study aimed to evaluate the quality of HVPG measurements at our centre before and after introduction of a standardised protocol, and the clinical relevance of the HVPG to variceal bleeding in cirrhotics.

METHODS HVPG measurements performed at Singapore General Hospital from 2005-2013 were retrospectively reviewed. Criteria for quality HVPG readings were triplicate readings, absence of negative pressure values and variability of $\leq 2 \mathrm{mmHg}$. The rate of variceal bleeding was compared in cirrhotics who achieved a HVPG response to pharmacotherapy (reduction of the HVPG to $<12 \mathrm{mmHg}$ or by $\geq 20 \%$ of baseline) and those who did not.

RESULTS 126 HVPG measurements were performed in 105 patients (mean age $54.7 \pm 11.4$ years; $55.2 \%$ men). $80 \%$ had liver cirrhosis and $20 \%$ had non-cirrhotic portal hypertension (NCPH). The mean overall HVPG was $13.5 \pm 7.2 \mathrm{mmHg}$, with a significant difference between the cirrhosis and NCPH groups $(p<0.001)$. The proportion of quality readings significantly improved after the protocol was introduced. HVPG response was achieved in $28(33.3 \%, n=84)$ cirrhotics. Nine had variceal bleeding over a median follow-up of 29 months. The rate of variceal bleeding was significantly lower in HVPG responders compared to nonresponders $(p=0.025)$.

CONCLUSION The quality of HVPG measurements in our centre improved after the introduction of a standardised protocol. A HVPG response can prognosticate the risk of variceal bleeding in cirrhotics.

Keywords: Asia, hepatic venous pressure gradient, quality, Singapore, variceal bleeding

\section{INTRODUCTION}

Portal hypertension is an important consequence in the natural history of liver cirrhosis and is recognised as an independent factor for survival. ${ }^{(1)}$ The severity of portal hypertension is directly related to the development of life-threatening consequences, including variceal bleeding, ascites, hepatic encephalopathy and hepatorenal syndrome. The quantitative estimation of portal hypertension via measurement of the hepatic venous pressure gradient (HVPG) is an accepted and validated method shown to have an important clinical impact on the management of patients with cirrhosis. ${ }^{(2,3)}$

Many publications have demonstrated the benefits of HVPG measurement in predicting the risk of variceal bleeding for both primary and secondary prophylaxis, and determining response to pharmacological therapy. ${ }^{(4,5)}$ However, HVPG measurement is an invasive procedure that requires expertise and technical training; as such, it is not routinely available in all hospitals. In recent years, there has been increasing acceptance of the role of the HVPG in the management of portal hypertension. The 2005 Baveno IV International Consensus Workshop on methodology of diagnosis and therapy in portal hypertension stated that "HVPG monitoring identifies patients with cirrhosis who will benefit from nonselective beta-blocker therapy in primary prophylaxis" but the "routine use of HVPG cannot be recommended". ${ }^{(6)}$ The HVPG has since demonstrated diagnostic, prognostic and therapeutic value in the management of liver cirrhosis. Specifically, it has been found that reduction of the HVPG to $<12 \mathrm{mmHg}$ or by $\geq 20 \%$ of the baseline value significantly reduces the risk of recurrent variceal bleeding and mortality, making the HVPG a valuable tool for the clinical management of cirrhosis. ${ }^{(5)}$ The most recent Baveno VI International Consensus Workshop in 2015 now recommends that "HVPG measurement is the gold standard method to assess the presence of clinically significant portal hypertension" ${ }^{\prime(7)}$ As the HVPG is used to guide important clinical decisions, it is crucial for the procedure to be performed in a standardised manner and that the results are accurate and reproducible. ${ }^{(8)}$

The HVPG is used in other areas of the world, but there have been no reports of its use in clinical practice in Southeast Asian countries. Although HVPG measurement has been performed at our centre since 2005, it was initially performed

${ }^{1}$ Department of Gastroenterology and Hepatology, Singapore General Hospital, ${ }^{2}$ Interventional Radiology Centre, Department of Diagnostic Radiology, Singapore General Hospital, ${ }^{3}$ Duke-NUS Graduate Medical School, ${ }^{4}$ Gleneagles Hospital, Singapore

Correspondence: Dr Jason Chang Pik Eu, Senior Consultant, Department of Gastroenterology and Hepatology, Singapore General Hospital, Outram Road, Singapore 169608. jason.chang@singhealth.com.sg 


\section{Table I. Standardised protocol for hepatic venous pressure gradient (HVPG) measurement.}

\begin{tabular}{|c|c|}
\hline 1. & All interventional radiologists involved in performing HVPG measurements are qualified and trained to perform HVPG. \\
\hline 2. & Appropriate recording scales for venous pressures are used instead of arterial pressure scales. \\
\hline 3. & $\begin{array}{l}\text { The transducer is fixed at the level of the right atrium in the midaxillary line for all patients and calibrated against known } \\
\text { external pressures. }\end{array}$ \\
\hline 4. & $\begin{array}{l}\text { Ultrasonography-guided puncture of the internal jugular vein is performed using a strict aseptic technique after infiltrating the } \\
\text { entry site with lidocaine. Position of the guidewire in the vein is confirmed by fluoroscopy. }\end{array}$ \\
\hline 5. & $\begin{array}{l}\text { A } 6 \text { French (Fr) sheath is inserted over the guidewire and is secured in the internal jugular vein, through which a } 4 \mathrm{Fr} \\
\text { Multipurpose A catheter (Cordis, Johnson and Johnson, Florida, USA) is advanced into the hepatic vein under fluoroscopic } \\
\text { guidance. The middle hepatic vein is preferentially cannulated, failing which the right hepatic vein is cannulated. }\end{array}$ \\
\hline 6. & The catheter is positioned no more than $2 \mathrm{~cm}$ into the hepatic vein for measurement of the free hepatic venous pressure (FHVP). \\
\hline 7. & $\begin{array}{l}\text { An occlusion } 5.5 \mathrm{Fr} \text { 'over-the-wire' Fogarty balloon catheter (Edwards Lifesciences, California, USA) is used for all wedged } \\
\text { hepatic venous pressure (WHVP) measurements unless deemed unnecessary by the operator. After advancing the catheter } \\
\text { distally into the appropriate position in the hepatic vein, the balloon is inflated. }\end{array}$ \\
\hline 8. & $\begin{array}{l}\text { After inflation of the balloon, } 5 \mathrm{~mL} \text { of contrast is injected and a venogram obtained to ensure total occlusion of the hepatic vein. } \\
\text { This step is essential to exclude the presence of intrahepatic venous-to-venous shunts that would result in underestimation of } \\
\text { the true WHVP. If total occlusion of the hepatic vein is not satisfactorily obtained, the balloon is deflated and the catheter is } \\
\text { repositioned to ensure adequate occlusion. }\end{array}$ \\
\hline 9. & Adequate time (at least 45-60 s) is allowed for stabilisation of readings before a final measurement is recorded. \\
\hline 10. & $\begin{array}{l}\text { The balloon is deflated and a second FHVP reading is measured. The balloon is then re-inflated and a second WHVP reading is } \\
\text { measured without changing the position of the balloon catheter. A total of three sets of FHVP and WHVP readings are measured. }\end{array}$ \\
\hline 11. & $\begin{array}{l}\text { If any of the repeat FHVP or WHVP readings differ by }>2 \mathrm{mmHg} \text {, the reading is discarded. The position of the catheter is } \\
\text { rechecked and the measurement is repeated. The balloon catheter may require flushing with saline to remove remnant contrast } \\
\text { material that may result in erroneous pressure readings. }\end{array}$ \\
\hline 12. & $\begin{array}{l}\text { In addition to the FHVP and WHVP readings, pressure measurements of the inferior vena cava (at the level of the hepatic veins), } \\
\text { right atrium and pulmonary artery are recorded. }\end{array}$ \\
\hline
\end{tabular}

by a small number of trained personnel without a specific protocol. Therefore, HVPG readings were often inconsistent and unstandardised, limiting their clinical applicability. In 2009, we initiated a multidisciplinary protocol to standardise HVPG measurement techniques in our centre based on recommended quality criteria. ${ }^{(8)}$ This study aimed to evaluate the quality of HVPG measurements performed in our centre before and after institution of a standardised protocol. In addition, we aimed to evaluate the clinical relevance of HVPG measurements to variceal bleeding in cirrhotics.

\section{METHODS}

Patients who underwent HVPG measurement at Singapore General Hospital, Singapore, from 2005-2013 were retrospectively identified from the computerised hospital database. Patient demographics and clinical data, including aetiology of liver disease, indication for HVPG measurement, dose of nonselective beta-blockers (NSBB) and episodes of variceal bleeding, were recorded in a standardised data form. HVPG data was systematically retrieved from the system, including measurements of the wedged hepatic venous pressure (WHVP), free hepatic venous pressure (FHVP), inferior vena cava (IVC) pressure at the level of the hepatic vein, and right atrial (RA) and pulmonary artery (PA) pressure. In patients with transjugular intrahepatic portosystemic shunts (TIPS), direct portal venous pressure, FHVP, IVC pressure and portosystemic gradient measurements were recorded.

Pressure measurements were performed using a catheter with a pressure transducer at the tip, which was passed under fluoroscopic guidance into either the middle or right hepatic vein.
This was accessed via the transjugular approach in all patients. Within the hepatic vein, the FHVP was recorded as the pressure reading when the catheter was free in the lumen of the hepatic vein (approximately $1-2 \mathrm{~cm}$ from the IVC). The catheter was advanced into the hepatic vein as far as possible and the WHVP was measured and recorded; it was then withdrawn and further pressure readings were taken in the IVC at the level of the hepatic vein, the RA and the PA (when indicated). All pressure readings were recorded in $\mathrm{mmHg}$. The HVPG was calculated using the formula: WHVP - FHVP = HVPG. There was no standardisation in the positioning of the transducer. Arterial pressure scales were sometimes used for measurement. Balloon catheters were not used for assessment of the WHVP.

A standardised HVPG protocol was introduced in 2009 in order to improve the quality of HVPG measurements at our centre (Table I). This was a collaborative multi-disciplinary effort including hepatologists and interventional radiologists involved in the management of patients with chronic liver diseases. The protocol incorporated the following standardised approach to HVPG measurements:

1. Patient selection - Indications for HVPG measurement were categorised as follows:

(a) Prognostication of variceal bleeding in liver cirrhosis - Cirrhotics with large varices who were on the maximal propranolol dosage (optimised to a resting heart rate of 60 beats per minute [bpm] and resting systolic blood pressure of $100 \mathrm{mmHg}$ ) were selected to undergo HVPG measurement to prognosticate for variceal bleeding risk. Mean propranolol dosage was $40 \mathrm{mg}$ TDS (three times a day; $120 \mathrm{mg}$ /day). Patients 
with a HVPG $<12 \mathrm{mmHg}$ were maintained on their propranolol dosage, whereas those with a HVPG $\geq 12 \mathrm{mmHg}$ either had their propranolol dose increased further (optimised to a resting heart rate of $55 \mathrm{bpm}$ ) or had nitrates added.

(b) Assessment of therapeutic response - In the subset of patients who had a baseline HVPG $\geq 12 \mathrm{mmHg}$, HVPG measurement was repeated after further optimisation of pharmacotherapy (either by increasing propranolol dosage or adding isosorbide mononitrate) to assess for response (defined as a reduction of the HVPG by $\geq 20 \%$ of baseline value and/or reduction in HVPG to $<12 \mathrm{mmHg}$ ). Patients were followed up to the end of the study to monitor for episodes of variceal bleeding.

(c) Diagnostic differentiation between cirrhotic and noncirrhotic portal hypertension $(\mathrm{NCPH})$ - Patients who presented with features of portal hypertension but did not have features suggestive of liver cirrhosis were selected for HVPG measurement and transjugular liver biopsy as part of a diagnostic protocol for the evaluation of $\mathrm{NCPH}$.

(d) Pre- and post-TIPS evaluation - HVPG measurements were routinely taken prior to the insertion of TIPS. For elective TIPS, the HVPG was measured as a separate procedure in the pre-TIPS evaluation to establish the baseline portosystemic gradient. In acute TIPS, the HVPG was measured immediately prior to the insertion of TIPS. The HVPG was routinely assessed in all post-TIPS patients on Day $5 \pm 2$ days to document the post-TIPS portosystemic gradient.

2. Standardisation of HVPG procedure - All interventional radiologists involved in performing HVPG measurements followed the standardised protocol detailed in Table I.

3. Reporting of HVPG results - The finalised HVPG results were reported using a standardised template that, in addition to RA, IVC and PA readings, required a complete description of triplicate WHVP, FHVP and HVPG values.

4. Post-HVPG care - All patients were provided with adequate compression and a pressure bandage over the puncture point in the neck. Analgesia (per oral paracetamol $1 \mathrm{~g}$ ) was given if required. Patients' vital signs were monitored every 15 minutes for the first two hours, every 30 minutes for the next two hours and every hour for the following two hours. Patients were monitored for the development of any neck haematoma.

The quality of HVPG measurements before and after the introduction of the protocol was compared to evaluate the effectiveness of the protocol in improving the quality of HVPG measurements in our centre. HVPG readings that fulfilled all three of the following criteria selected for this study were considered quality HVPG readings: (a) Measurements should be repeated at least three times (i.e. in triplicate) in order to ensure reproducibility. (b) There should not be any negative pressure readings. Such readings indicate improper placement of the transducer, resulting in erroneous measurements. (c) For a
Table II. Demographics of patients included in the study $(n=105)$.

\begin{tabular}{|c|c|}
\hline Characteristic & No. (\%) \\
\hline 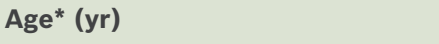 & $54.7 \pm 11.4$ \\
\hline \multicolumn{2}{|l|}{ Sex } \\
\hline Male & $58(55.2)$ \\
\hline Female & $47(44.8)$ \\
\hline \multicolumn{2}{|l|}{ Ethnicity } \\
\hline Chinese & $85(81.0)$ \\
\hline Malay & $8(7.6)$ \\
\hline Indian & $8(7.6)$ \\
\hline Others & $4(3.8)$ \\
\hline \multicolumn{2}{|l|}{ Aetiology of liver disease } \\
\hline Hepatitis B & $30(28.6)$ \\
\hline Hepatitis C & $5(4.8)$ \\
\hline Nonalcoholic steatohepatitis & $15(14.3)$ \\
\hline Alcoholism & $15(14.3)$ \\
\hline Cryptogenic cirrhosis & $13(12.4)$ \\
\hline Autoimmune cirrhosis (AlH/PBC) & $6(5.7)$ \\
\hline $\mathrm{NCPH}$ & $21(20.0)$ \\
\hline \multicolumn{2}{|l|}{ Indication for HVPG measurements } \\
\hline Diagnostic (cirrhosis vs. NCPH) & $44(41.9)$ \\
\hline Assess response to beta-blocker & $46(43.8)$ \\
\hline Pre-TIPS assessment & 15 (14.3) \\
\hline \multicolumn{2}{|l|}{ HVPG studies performed per patient } \\
\hline 1 & $90(85.7)$ \\
\hline 2 & $9(8.6)$ \\
\hline 3 & $6(5.7)$ \\
\hline \multicolumn{2}{|l|}{ HVPG* (mmHg) } \\
\hline Overall & $13.5 \pm 7.2$ \\
\hline Cirrhosis group & $15.8 \pm 6.2$ \\
\hline NCPH group & $5.3 \pm 3.9$ \\
\hline
\end{tabular}

*Data presented as mean \pm standard deviation. AlH: autoimmune hepatitis; HVPG: hepatic venous pressure gradient; $\mathrm{NCPH}$ : non-cirrhotic portal hypertension; PBC: primary biliary cirrhosis; TIPS: transjugular intrahepatic portosystemic shunts

meaningful median value to be selected, measurements should be consistent and should not differ by more than $2 \mathrm{mmHg}$.

Clinical outcome was based on available hospital records up to 31 December 2013. Hospital admissions for variceal bleeding, ascites, hepatic encephalopathy and development of hepatocellular carcinoma were recorded, as well as complications from the HVPG procedure and patient mortality during the study period. The main clinical outcome in this study was variceal bleeding occurring after baseline HVPG measurement was performed. The median follow-up period was 29 (range 1-104) months.

Data analysis was performed using IBM SPSS Statistics version 19.0 (IBM Corp, Armonk, NY, USA). Mean values (expressed as mean \pm standard deviation) of the HVPG were tabulated for the various groups of patients and compared using Student's $t$-test. Proportions were compared using chi-square test. A p-value $<0.05$ indicated statistical significance. The study protocol was approved by the hospital's Centralised Institutional Review Board. 


\section{RESULTS}

A total of 126 HVPG measurements from 105 patients were taken between 2005 and 2013 (Table II). The mean patient age was $54.7 \pm 11.4$ years and $55.2 \%(n=58)$ of the patients were men. $81.0 \%$ of the patients were Chinese, $7.6 \%$ were Malay and $7.6 \%$ were Indian. The aetiology of liver disease was liver cirrhosis in $80.0 \%$ of the patients and $\mathrm{NCPH}$ in the remaining $20.0 \%$. The aetiologies of liver cirrhosis included chronic hepatitis B (28.6\%), alcoholism (14.3\%), nonalcoholic steatohepatitis $(14.3 \%)$, chronic hepatitis C $(4.8 \%)$, cryptogenic cirrhosis $(12.4 \%)$ and autoimmune liver disorders (5.7\%). 90 patients (85.7\%) had a single HVPG measurement and 15 (14.3\%) had repeat HVPG measurements. The mean HVPG for all patients was $13.5 \pm 7.2 \mathrm{mmHg}$ and was significantly different between patients with liver cirrhosis and those with $\mathrm{NCPH}$ $(15.8 \pm 6.2 \mathrm{mmHg}$ vs. $5.3 \pm 3.9 \mathrm{mmHg} ; \mathrm{p}<0.001)$. Indications for HVPG measurement were: assessment of response to NSBB therapy $(43.8 \%)$, diagnosis of portal hypertension $(41.9 \%)$ and pre-TIPS assessment (14.3\%).

The number of HVPG measurements performed per year increased after the implementation of the standardised protocol in 2009. Between 2005 and 2008, 18 (17.1\%) HVPG measurements were performed, whereas 87 (82.9\%) were performed from 2009 to 2013 after the introduction of the standardised protocol. Quality HVPG readings were obtained in $59.0 \%$ of the entire cohort (Table III). After introduction of the standardised protocol, the proportion of quality readings improved significantly from $5.6 \%$ to $70.1 \%$ ( $p<0.001$ ). Of the three defining criteria for quality readings, there was a significant improvement in the number of triplicate readings and number of readings that did not differ by $>2 \mathrm{mmHg}$, but there was no significant difference in the presence of negative pressure readings.

We analysed the 84 patients with cirrhosis to evaluate the difference in the occurrence of variceal bleeding in those who achieved a target HVPG response, defined as a HVPG $<12 \mathrm{mmHg}$ in patients who were on optimal doses of NSBB, and/or $\geq 20 \%$ reduction from baseline in patients with repeat HVPG measurements after optimisation of pharmacotherapy. $9(10.7 \%)$ patients had variceal bleeding during the follow-up period (Table IV). The rate of variceal bleeding was significantly higher in those who failed to achieve the target HVPG response (16.1\%) compared to those who achieved a HVPG response $(0 \%)$. None of the cirrhotics with a HVPG $<12 \mathrm{mmHg}$ had variceal bleeding. 15 patients had repeat HVPG measurements after optimisation of pharmacotherapy following a baseline HVPG measurement. 4 (44.4\%) of the nine patients who failed to achieve $20 \%$ reduction of the baseline HVPG had variceal bleeding. In contrast, no variceal bleeding occurred in the six patients who had reduction of the HVPG of $\geq 20 \%$ from the baseline.

No significant complications were encountered in the 126 HVPG studies performed during the study period. Specifically, no patients developed significant neck haematomas, inadvertent carotid punctures or significant cardiac arrhythmias.
Table III. Proportion of quality hepatic venous pressure gradient measurements before and after the standardised protocol.

\begin{tabular}{|c|c|c|c|}
\hline Fulfilled criteria & $\begin{array}{l}\text { Before protocol } \\
(n=18)(\%)\end{array}$ & $\begin{array}{l}\text { After protocol } \\
(n=87)(\%)\end{array}$ & p-value \\
\hline Triplicate readings & $2(11.1)$ & $63(72.4)$ & $<0.001$ \\
\hline $\begin{array}{l}\text { Readings do not differ } \\
\text { by }>2 \mathrm{mmHg}\end{array}$ & $1(5.6)$ & $61(70.1)$ & $<0.001$ \\
\hline $\begin{array}{l}\text { Absence of negative } \\
\text { pressure readings }\end{array}$ & $16(88.9)$ & $82(94.3)$ & NS \\
\hline All three quality criteria & $1(5.6)$ & $61(70.1)$ & $<0.001$ \\
\hline
\end{tabular}

NS: not significant

Table IV. Rate of variceal bleeding in cirrhotics after hepatic venous pressure gradient (HVPG) optimisation $(n=84)$.

\begin{tabular}{|c|c|c|}
\hline Characteristic & $\begin{array}{c}\text { No. of patients } \\
\text { with variceal } \\
\text { bleeding (\%) }\end{array}$ & p-value \\
\hline HPVG response & & 0.03 \\
\hline Yes $(n=28)$ & 0 & \\
\hline No $(n=56)$ & $9(16.1)$ & \\
\hline HPVG & & 0.04 \\
\hline$<12 \mathrm{mmHg}(\mathrm{n}=24)$ & 0 & \\
\hline$\geq 12 \mathrm{mmHg}(\mathrm{n}=60)$ & $9(15.0)$ & \\
\hline Reduction from baseline $(n=15)$ & & 0.07 \\
\hline$\geq 20 \%(n=6)$ & 0 & \\
\hline$<20 \%(n=9)$ & $4(44.4)$ & \\
\hline
\end{tabular}

\section{DISCUSSION}

We have shown that the implementation of a standardised protocol significantly improved the quality of HVPG studies performed in our centre. This supports the recommendations by Groszmann and Wongcharatrawee ${ }^{(8)}$, and Kumar et $\mathrm{al}^{(9)}$, who advocate the importance of proper HVPG technique in order for meaningful clinical decisions to be made based on these measurements. Prior to implementation of the standardised protocol, there was wide variation in the quality of HVPG measurements at our centre; many patients did not have repeat measurements and more than half had variations in repeat readings of $>2 \mathrm{mmHg}$. The final reports of many HVPG studies were incomplete, making interpretation of the data difficult. With the implementation of a standardised protocol, we demonstrated the ability to consistently obtain quality HVPG readings at our centre. Our study also provides evidence to support the role of the HVPG in the clinical management of variceal bleeding in cirrhotics. Reliable HVPG readings enable clinicians to prognosticate bleeding risk in responders and nonresponders to NSBB, and manage their patients appropriately.

The HVPG results in our study are compatible with those reported in the literature. The mean HVPG in our cirrhotic cohort was $15.8 \pm 6.2 \mathrm{mmHg}$. This is similar to the studies published by Kim et $\mathrm{al}^{(10)}$ and Garg et al, ${ }^{(11)}$ which reported a mean HVPG of $15.6 \pm 5.1 \mathrm{mmHg}$ and median HVPG of 16 (range 12-30) $\mathrm{mmHg}$, respectively. Despite a small sample size, our study demonstrated a significant difference $(p=0.04)$ in the risk of variceal bleeding in cirrhotics with a HVPG $\geq 12 \mathrm{mmHg}$ compared with those 
with a HVPG $<12 \mathrm{mmHg}$. The incidence of variceal bleeding in the former group was $15.0 \%$ over a median follow-up period of 2.5 years. In the latter group with a HVPG $<12 \mathrm{mmHg}$, no patient had variceal bleeding, confirming the observation by Groszmann. ${ }^{(12)}$ The $12 \mathrm{mmHg}$ cut-off value is useful to guide management, providing prognostic and therapeutic benefits including titrating the dosage of beta-blockers. ${ }^{(13,14)}$

HVPG measurement is a useful diagnostic tool in the evaluation of portal hypertension and is useful in differentiating between cirrhotic and non-cirrhotic aetiologies. Although the clinical presentation of $\mathrm{NCPH}$ is similar to cirrhosis with splenomegaly, thrombocytopenia, large varices and nodularity of the liver on imaging, a normal HVPG reading suggests a diagnosis of $\mathrm{NCPH}$, in contrast to the elevated HVPG found in liver cirrhosis. ${ }^{(15,16)}$ In our study cohort, patients with NCPH had a normal mean HVPG of $5.3 \pm 3.9 \mathrm{mmHg}$. This demonstrates the clinical utility of the HVPG in differentiating the classical sinusoidal portal hypertension seen in cirrhosis from the pre- or post-hepatic portal hypertension associated with non-cirrhotic aetiologies of portal hypertension. Coupled with transjugular liver biopsy, HVPG measurement is an essential diagnostic tool and should be an integral part of the routine evaluation of patients with portal hypertension in whom a non-cirrhotic aetiology is suspected. ${ }^{(17)}$

International consensus guidelines now recommend the routine use of the HVPG for prognostic and therapeutic indications in primary prophylaxis of variceal bleeding. ${ }^{(7,18)}$ For the reduction of mortality, a therapeutic target of a decrease in the HVPG of $\geq 20 \%$ from baseline or to $<12 \mathrm{mmHg}$ after optimised treatment with NSBB is clinically relevant, and represents the current standard of care. ${ }^{(5)}$ In order to optimise the management of our patients with portal hypertension, it is important for us to be able to perform HVPG measurements in a safe and reliable manner. The use of HVPG-based response guided therapy, otherwise termed the 'a la carte' approach, has been shown to reduce variceal bleeding rates in responders to pharmacologic therapy to $10 \%$ over two years. ${ }^{(19)}$

The HVPG also plays an important role in differentiating responders to NSBB from nonresponders. It has been suggested that an acute HVPG response (by a reduction in the HVPG of at least $10 \%$ ) to intravenous propranolol can effectively identify responders to NSBB. ${ }^{(20)}$ This strategy is useful in identifying the nonresponders, for whom endoscopic band ligation should be recommended. This will reduce the rate of variceal bleeding, which is estimated to be approximately $30 \%$ in NSBB nonresponders.

In patients presenting with acute variceal bleeding, early HVPG measurements have been shown to identify a subgroup of patients who are at high risk of early rebleeding. A HVPG $\geq 20 \mathrm{mmHg} 24$ hours after an episode of acute variceal bleeding is predictive of treatment failure and increased mortality. ${ }^{(21)}$ Early TIPS in these high-risk patients has been shown to reduce mortality risk. ${ }^{(22)}$ The HVPG has also been shown to be useful in predicting prognosis and survival in cirrhotics. ${ }^{(23)}$ It also has clinical value in preoperative risk assessment of cirrhotics undergoing hepatic resection for hepatocellular carcinoma. ${ }^{(24)}$
The implementation of a standardised protocol enabled our centre to provide quality HVPG readings in more than $70 \%$ of procedures. In the remaining $30 \%$ of cases in which quality readings were not obtained, there was a significant difference in the measurement of HVPG readings in triplicate based on the indication for HVPG. In patients who had HVPG measured for pre-TIPS assessment, only $53.8 \%$ of cases had triplicate readings. In contrast, $75.7 \%$ of patients undergoing HVPG measurement for non-TIPS indications had triplicate readings according to the prescribed protocol. In patients undergoing emergency TIPS for acute variceal bleeding, time is often of the essence. In these unstable patients, the priority is often to deploy and inflate TIPS as soon as possible in order to reduce the portal pressure and stop the variceal bleeding. In such situations, the procedurist may not take the additional time to measure pressure readings in triplicate.

Despite its retrospective nature, the results of this study provide objective information about the feasibility and quality of HVPG measurements performed at our centre. To date, there is no published data on HVPG procedures in Singapore. Even within the Southeast Asian region, there is little information about the ability to provide reliable and accurate HVPG measurements. We believe it is important for practising physicians to be updated on the feasibility of HVPG-guided pharmacotherapy in the management of portal hypertension in our region. Our study provides some evidence that HVPG-guided therapy improves clinical outcome in cirrhotics. However, a prospective study of the 'a la carte' treatment based on baseline and repeat HVPG measurements is required to provide conclusive evidence of significant improvement in clinical outcome in local patients. In addition, our study provides evidence that HVPG measurement is a safe and well-tolerated procedure with minimal patient discomfort.

In conclusion, our centre possesses the adequate resources and expertise to perform HVPG measurements of good quality that are in keeping with international recommendations. We recommend that HVPG measurements should be routinely used for prognostic and therapeutic indications in the management of patients with portal hypertension in Singapore.

\section{ACKNOWLEDGEMENTS}

The authors would like to acknowledge Austin Htoo Myint Maung, Manish Taneja, Sateesh, Lin Shueh En, Khoo Li Ser, Yeow Tow Nan, Terence Teo, Jonathan Robertson, Shaun Chen, Ankur Patel and others for their participation in performing HVPG measurements in accordance with the prescribed protocol, and members of the Department of Gastroenterology and Hepatology, Singapore General Hospital, Singapore, for contributing patients to the study.

\section{REFERENCES}

1. Armonis A, Patch D, Burroughs A. Hepatic venous pressure measurement: an old test as a new prognostic marker in cirrhosis? Hepatology 1997; 25:245-8.

2. Feu F, García-Pagán JC, Bosch J, et al. Relation between portal pressure response to pharmacotherapy and risk of recurrent variceal haemorrhage in patients with cirrhosis. Lancet 1995; 346:1056-9. 
3. Bosch J, Abraldes JG, Berzigotti A, García-Pagan JC. The clinical use of HVPG measurements in chronic liver disease. Nat Rev Gastroenterol Hepatol 2009; 6:573-82.

4. Abraldes JG, Tarantino I, Turnes J, et al. Hemodynamic response to pharmacological treatment of portal hypertension and long-term prognosis of cirrhosis. Hepatology 2003; 37:902-8.

5. D'Amico G, Garcia-Pagan JC, Luca A, Bosch J. Hepatic vein pressure gradient reduction and prevention of variceal bleeding in cirrhosis: a systematic review. Gastroenterology 2006; 131:1611-24.

6. de Franchis R. Evolving consensus in portal hypertension. Report of the Baveno IV consensus workshop on methodology of diagnosis and therapy in portal hypertension. J Hepatol 2005; 43:167-76.

7. de Franchis R; Baveno VI Faculty. Expanding consensus in portal hypertension: Report of the Baveno VI consensus workshop: Stratifying risk and individualizing care for portal hypertension. J Hepatol 2015; 63:743-52.

8. Groszmann RJ, Wongcharatrawee $\mathrm{S}$. The hepatic venous pressure gradient: anything worth doing should be done right. Hepatology 2004; 39:280-2.

9. Kumar A, Sharma P, Sarin SK. Hepatic venous pressure gradient measurement: time to learn! Indian J Gastroenterol 2008; 27:74-80.

10. Kim MY, Baik SK, Suk KT, et al. [Measurement of hepatic venous pressure gradient in liver cirrhosis: relationship with the status of cirrhosis, varices, and ascites in Korea]. Korean J Hepatol 2008; 14:150-8. Korean.

11. Garg H, Kumar A, Garg V, et al. Hepatic and systemic hemodynamic derangements predict early mortality and recovery in patients with acuteon-chronic liver failure. J Gastroenterol Hepatol 2013; 28:1361-7.

12. Groszmann RJ, Bosch J, Grace ND, et al. Hemodynamic events in a prospective randomized trial of propranolol versus placebo in the prevention of a first variceal hemorrhage. Gastroenterology 1990; 99:1401-7.

13. Garcia-Tsao G, Groszmann RJ, Fisher RL, et al. Portal pressure, presence of gastroesophageal varices and variceal bleeding. Hepatology; 5:419-24.

14. Addley J, Tham TC, Cash WJ. Use of portal pressure studies in the management of variceal haemorrhage. World J Gastrointest Endosc 2012; $4: 281-9$

15. Khanna R, Sarin SK. Non-cirrhotic portal hypertension - diagnosis and management. J Hepatol 2014; 60:421-41.

16. Seijo S, Reverter E, Miquel R, et al. Role of hepatic vein catheterisation and transient elastography in the diagnosis of idiopathic portal hypertension. Dig Liver Dis 2012; 44:855-60.

17. Patel A, Gogna A, Irani FG, et al. Single centre experience of transjugular liver biopsy in 152 patients. Ann Acad Med Singapore 2014; 43:160-5.

18. Garcia-Tsao G, Sanyal AJ, Grace ND, Carey W; Practice Guidelines Committee of the American Association for the Study of Liver Diseases; Practice Parameters Committee of the American College of Gastroenterology. Prevention and management of gastroesophageal varices and variceal hemorrhage in cirrhosis. Hepatology 2007; 46:922-38.

19. Bureau C, Péron JM, Alric L, et al. "A La Carte" treatment of portal hypertension: Adapting medical therapy to hemodynamic response for the prevention of bleeding. Hepatology 2002; 36:1361-6.

20. La Mura V, Abraldes JG, Raffa S, et al. Prognostic value of acute hemodynamic response to i.v. propranolol in patients with cirrhosis and portal hypertension. J Hepatol 2009; 51:279-87.

21. Moitinho E, Escorsell A, Bandi JC, et al. Prognostic value of early measurements of portal pressure in acute variceal bleeding. Gastroenterology 1999; 117:626-31.

22. Monescillo A, Martínez-Lagares F, Ruiz-del-Arbol L, et al. Influence of portal hypertension and its early decompression by TIPS placement on the outcome of variceal bleeding. Hepatology 2004; 40:793-801.

23. Ripoll C, Groszmann R, Garcia-Tsao G, et al; Portal Hypertension Collaborative Group. Hepatic venous pressure gradient predicts clinical decompensation in patients with compensated cirrhosis. Gastroenterology 2007; 133:481-8.

24. Stremitzer S, Tamandl D, Kaczirek K, et al. Value of hepatic venous pressure gradient measurement before liver resection for hepatocellular carcinoma. Br J Surg 2011; 98:1752-8. 